\title{
SOME REMARKS ON THE HOMOLOGY OF MODULI SPACE OF INSTANTONS WITH INSTANTON NUMBER 2
}

\author{
YASUHIKO KAMIYAMA \\ (Communicated by Frederick R. Cohen) \\ Dedicated to Professor Akio Hattori on his sixtieth birthday
}

\begin{abstract}
Let $M_{2}$ be the framed moduli space of SU(2) instantons with instanton number 2. By combining the results of Boyer and Mann and the results of Hattori, we determine the structure of $H^{*}\left(M_{2} ; Z_{2}\right)$.
\end{abstract}

\section{INTRODUCTION}

We shall denote by $M_{k}$ the framed moduli space of $\mathrm{SU}(2)$ instantons with instanton number $c_{2}=-k$. Recently Boyer and Mann [1] constructed homology operations on $M_{k}$ for all $k$ and thus constructed new homology classes in $H_{*}\left(M_{k} ; \mathbf{Z}_{p}\right)$. In the case $k=p=2$, the result is as follows.

Theorem 1 [1]. The elements of $H_{*}\left(M_{2} ; \mathbf{Z}_{2}\right)$ constructed by Boyer and Mann are given by the following table:

\begin{tabular}{c|c|c|c|c}
$q$ & 1 & 2 & \multicolumn{2}{c}{3} \\
\hline$H_{q}\left(M_{2} ; \mathbf{Z}_{2}\right)$ & $z_{1} *[1]$ & $z_{1}^{2} z_{2} *[1]$ & $Q_{1}\left(z_{1}\right) z_{2} * z_{1}$ & $z_{3} *[1]$ \\
& & 4 & 5 \\
\cline { 2 - 6 } & $Q_{2}\left(z_{1}\right) z_{2}^{2} z_{3} * z_{1}$ & $Q_{1}\left(z_{2}\right) z_{3} * z_{2} Q_{3}\left(z_{1}\right)$ \\
& & 7 & 8 & 9 \\
\hline$z_{3}^{2} Q_{2}\left(z_{2}\right)$ & $Q_{3}\left(z_{2}\right) Q_{1}\left(z_{3}\right)$ & $Q_{2}\left(z_{3}\right)$ & $Q_{3}\left(z_{3}\right)$
\end{tabular}

In another direction Hattori [4] completely determined the homotopy type of $M_{2}$ and as a result computed $H^{*}\left(M_{2} ; \mathbf{Z}\right)$ and $H^{*}\left(M_{2} ; \mathbf{Z}_{2}\right)$. The results are as follows.

Received by the editors December 5, 1989.

1980 Mathematics Subject Classification (1985 Revision). Primary 58E15; Secondary 55S12.

Key words and phrases. Instantons, loop run, Dyer-Lashof operations. 
Theorem 2 [4]. The cohomology groups of $M_{2}$ with $\mathbf{Z}$ coefficients are given by the following table:

\begin{tabular}{c|ccccccccc}
$q$ & 1 & 2 & 3 & 4 & 5 & 6 & 7 & 8 & 9 \\
\hline$H^{q}\left(M_{2} ; \mathbf{Z}\right)$ & 0 & $\mathbf{Z}_{2}$ & $\mathbf{Z}_{2}$ & $\mathbf{Z}_{3} \oplus \mathbf{Z}_{4}$ & $\mathbf{Z}_{2}$ & $\mathbf{Z}_{2}$ & $\mathbf{Z} \oplus \mathbf{Z}_{2}$ & 0 & $\mathbf{Z}_{2}$ \\
\hline generators & & $\beta$ & $\gamma$ & $p^{*} z \delta$ & $\beta \gamma$ & $\beta \delta$ & $\xi$ & $\gamma \delta$ &
\end{tabular}

Theorem 3 [4]. The cohomology groups of $M_{2}$ with $\mathbf{Z}_{2}$ coefficients are given by the following table:

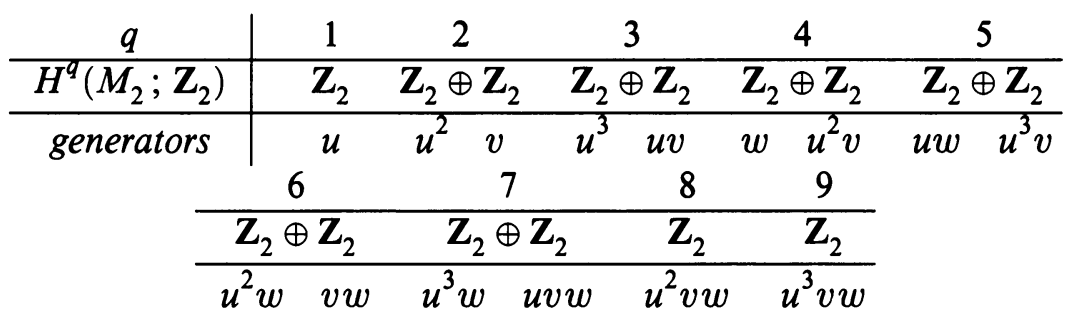

The choice of the elements $v$ and $w$ will be specified later.

In this paper we combine these results to obtain further homological information about $M_{2}$.

\section{MAIN Results}

We first study the following problem. Do the elements of Theorem 1 generate $H_{*}\left(M_{2} ; \mathbf{Z}_{2}\right)$ ?

Proposition 1. The elements of Theorem 1 generate $H_{*}\left(M_{2} ; \mathbf{Z}_{2}\right)$ and the following relations hold:

(1) $Q_{1}\left(z_{1}\right)+z_{2} * z_{1}+z_{3} *[1]=0$.

(2) $Q_{2}\left(z_{1}\right)=z_{3} * z_{1}$.

(3) $Q_{1}\left(z_{2}\right)+z_{3} * z_{2}+Q_{3}\left(z_{1}\right)=0$.

Proof. Let $\mathscr{C}_{2}$ be the orbit space of SU(2) connections with instanton number 2 by the action of the based gauge group and let $i: M_{2} \rightarrow \mathscr{C}_{2}$ be the inclusion.

Direct computations show that each element of Theorem 1 is nontrivial in $H_{*}\left(\mathscr{C}_{2} ; \mathbf{Z}_{2}\right)$ and differs in $H_{*}\left(\mathscr{C}_{2} ; \mathbf{Z}_{2}\right)$ except for

$$
i_{*} Q_{2}\left(z_{2}\right)=i_{*} z_{3} * z_{1} \text {. }
$$

Therefore by using Theorem 3 we see that the elements of Theorem 1 generate $H_{*}\left(M_{2} ; \mathbf{Z}_{2}\right)$ and there must be one relation for $q=3,4,5$.

But [1, Proposition 9.10] shows that there are the following relations.

(i) $i_{*}\left(Q_{1}\left(z_{1}\right)+z_{2} * z_{1}+z_{3} *[1]\right)=0$.

(ii) $i_{*}\left(Q_{2}\left(z_{1}\right)+z_{3} * z_{1}\right)=0$.

Using Cartan formula and Adem relation [2] we also see the following relation. 
(iii) $i_{*}\left(Q_{1}\left(z_{2}\right)+z_{3} * z_{2}+Q_{3}\left(z_{1}\right)\right)=0$.

Now by using Theorem 3 we see that the relations (i)-(iii) imply the relations (1)-(3) in Proposition 1.

Next we shall study the Kronecker products of elements of Theorems 1 and 3. On account of Proposition 1 we can take a basis of $H_{q}\left(M_{2} ; \mathbf{Z}_{2}\right)$ for $q=3,4,5$ as follows:

$$
\begin{array}{lll}
q=3 & Q_{1}\left(z_{1}\right) & z_{2} * z_{1} \\
q=4 & z_{2}^{2} & z_{3} * z_{1} \\
q=5 & Q_{1}\left(z_{2}\right) & z_{3} * z_{2}
\end{array}
$$

Theorem 4. The Kronecker products of elements of Theorems 1 and 3 are given by the following table:

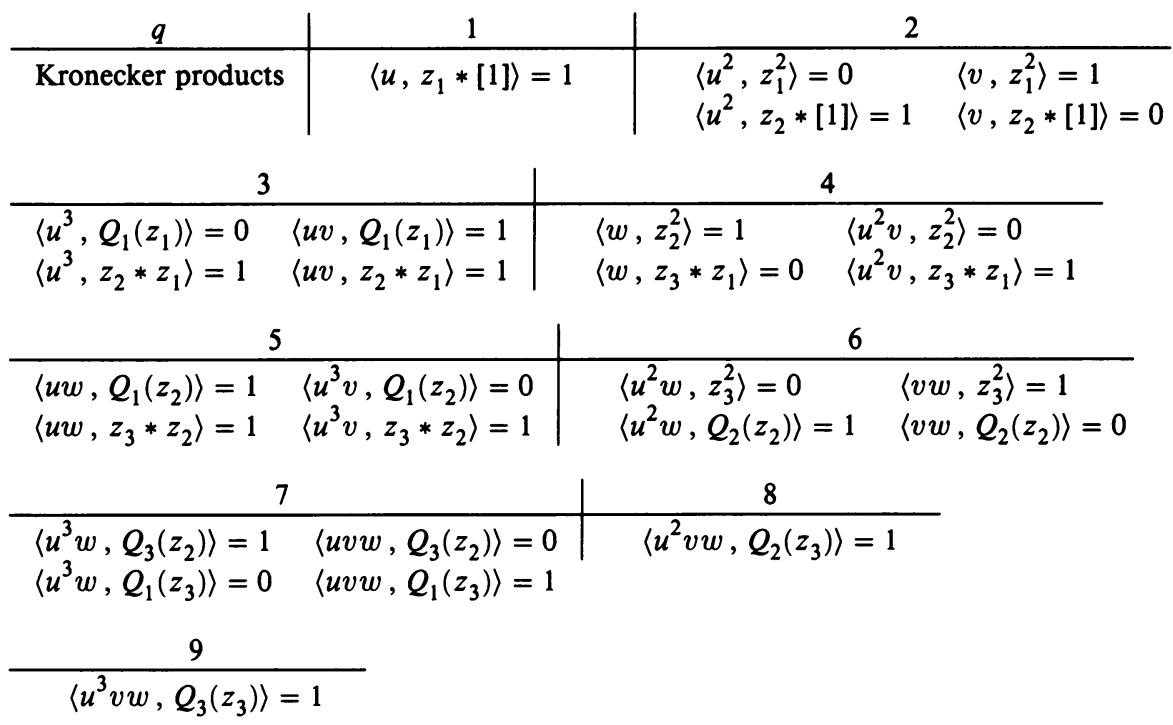

In the above table we define $v$ by

$$
\left\langle v, z_{1}^{2}\right\rangle=1, \quad\left\langle v, z_{2} *[1]\right\rangle=0 .
$$

Note that

$$
\left\langle u^{2}, z_{1}^{2}\right\rangle=0, \quad\left\langle u^{2}, z_{2} *[1]\right\rangle=1
$$

We define $w$ by

$$
\left\langle w, z_{2}^{2}\right\rangle=1, \quad\left\langle w, z_{3} * z_{1}\right\rangle=0
$$

Note that

$$
\left\langle u^{2} v, z_{2}^{2}\right\rangle=0, \quad\left\langle u^{2} v, z_{3} * z_{1}\right\rangle=1
$$


Proof. Let $\Delta: M_{k} \rightarrow M_{k} \times M_{k}$ be the diagonal. Then we can easily show the following relations.

$$
\begin{aligned}
& \Delta_{*} z_{1}=z_{1} \otimes[1]+[1] \otimes z_{1}, \\
& \Delta_{*} z_{2}=z_{2} \otimes[1]+z_{1} \otimes z_{1}+[1] \otimes z_{2}, \\
& \Delta_{*} z_{3}=z_{3} \otimes[1]+z_{2} \otimes z_{1}+z_{1} \otimes z_{2}+[1] \otimes z_{3} .
\end{aligned}
$$

The following relation is known in [2].

$$
\Delta_{*} Q_{j}(a)=\sum_{r, s} Q_{j-r}\left(a_{s}^{\prime}\right) \otimes Q_{r}\left(a_{s}^{\prime \prime}\right)
$$

where $\Delta_{*} a=\sum_{s} a_{s}^{\prime} \otimes a_{s}^{\prime \prime}$. Theorem 4 easily follows from these results.

Next we shall study the integral classes. On account of Theorem 2 there exists an element $\sigma$ that generates $\mathbf{Z}_{4}$ in $H_{3}\left(M_{2} ; \mathbf{Z}\right)$ and there exists an element $\tau$ that generates $\mathbf{Z}$ in $H_{7}\left(M_{2} ; \mathbf{Z}\right)$. Let

$$
j_{*}: H_{*}\left(M_{2} ; \mathbf{Z}\right) \rightarrow H_{*}\left(M_{2} ; \mathbf{Z}_{2}\right)
$$

be $\bmod 2$ reduction.

We shall study $j_{*} \sigma$ and $j_{*} \tau$.

Theorem 5. The following relations hold.

$$
\begin{gathered}
j_{*} \sigma=z_{3} *[1], \\
j_{*} \tau=Q_{3}\left(z_{2}\right) .
\end{gathered}
$$

Proof. Let $\left\{E_{*}^{r}\right\}$ be the mod 2 homology Bockstein spectral sequence of $M_{2}$. The following Nishida relation is known in [2].

$$
\beta Q^{j}(a)=(j-1) Q^{j-1}(a),
$$

where $\beta$ is the Bockstein operation.

By using the Nishida relation we compute $E_{*}^{2}$ as follows.

\begin{tabular}{c|ccccccccc}
$q$ & 1 & 2 & 3 & 4 & 5 & 6 & 7 & 8 & 9 \\
\hline$E_{q}^{2}$ & 0 & 0 & $z_{3} *[1]$ & $z_{2}^{2}$ & 0 & 0 & $Q_{3}\left(z_{2}\right)$ & 0 & 0
\end{tabular}

From this table Theorem 5 follows.

Next as an application of Proposition 1 and Theorem 4, we prove the following theorem.

Theorem 6. The elements of Theorem 2 satisfy the following relations:

(1) $\beta^{2}=2 \delta$,

(2) $\delta^{2}=0$,

(3) $\gamma^{2}=\beta \delta$.

Note that Theorem 6 completely determines the ring structure of $H^{*}\left(M_{2} ; \mathbf{Z}\right)$. 
Proof. (1) is shown in [4].

As $H^{8}\left(M_{2} ; \mathbf{Z}\right)=0$ holds, (2) follows.

We shall prove (3). Let

be $\bmod 2$ reduction.

$$
j_{*}: H^{*}\left(M_{2} ; \mathbf{Z}\right) \rightarrow H^{*}\left(M_{2} ; \mathbf{Z}_{2}\right)
$$

All we have to show to prove (3) is $j_{*} \gamma^{2} \neq 0$. Let $u, v, w$ be elements in Theorem 3. Either $j_{*} \gamma=u^{3}$ or $u v$ or $u^{3}+u v$ holds. We shall show that $j_{*} \gamma=u^{3}$ cannot occur. Assertion 1. The following relations hold.

$$
u^{4}=0, \quad v^{2}=w .
$$

In fact, in the same way as the proof of Theorem 4, we see the following Kronecker products.

$$
\begin{array}{ll}
\left\langle u^{4}, z_{2}^{2}\right\rangle=0, & \left\langle u^{4}, z_{3} * z_{1}\right\rangle=0, \\
\left\langle v^{2}, z_{2}^{2}\right\rangle=1, & \left\langle v^{2}, z_{3} * z_{1}\right\rangle=0 .
\end{array}
$$

Assertion 2. The following relation holds.

$$
j_{*} \beta=u^{2} \text {. }
$$

In fact, the following holds.

$$
j_{*} \beta=S q^{1} u=u^{2} \text {. }
$$

Now suppose $j_{*} \gamma=u^{3}$. The table in Theorem 2 shows that

$$
j_{*}(\beta \gamma) \neq 0 \text {. }
$$

But from Assertions 1 and 2 we have

$$
j_{*}(\beta \gamma)=\left(j_{*} \beta\right)\left(j_{*} \gamma\right)=u^{2} u^{3}=0 .
$$

This is a contradiction. Therefore either $j_{*} \gamma=u v$ or $u^{3}+u v$ holds. Anyway

$$
\left(j_{*} \gamma\right)^{2}=u^{2} v^{2}=u^{2} w \neq 0
$$

This completes the proof of $(3)$.

Remark. In [4], whether $\gamma^{2}=0$ or not is left unknown.

Now by using the above results, we can completely determine $H^{*}\left(M_{2} ; \mathbf{Z}_{2}\right)$.

Theorem 7. $H^{*}\left(M_{2} ; \mathbf{Z}_{2}\right)=\mathbf{Z}_{2}[u, v] /\left(u^{4}, v^{4}\right)$ and $S q^{1} v=u v$ hold. Note that the $\mathscr{A}(2)$-module structure of $H^{*}\left(M_{2} ; \mathbf{Z}_{2}\right)$ is completely determined.

Proof. The ring structure follows from Theorem 3 and Assertion 1 in Theorem 6. By using Theorem 4 and the following Kronecker products we can easily prove $S q^{1} v=u v$.

$$
\left\langle S q^{1} v, Q_{1}\left(z_{1}\right)\right\rangle=1, \quad\left\langle S q^{1} v, z_{2} * z_{1}\right\rangle=1 .
$$

\section{APPENDIX}

The proof of Proposition 9.5 seems incomplete in [1]. By using Theorem 3, we shall give an explicit proof of this proposition. 
Proposition 9.5 [1]. $z_{i} *[1]=Q_{i}[1]$ for $i=1,2,3$.

Proof. The proof of $z_{1} *[1]=Q_{1}[1]$ is given in [1].

(i) Proof of $z_{2} *[1]=Q_{2}[1]$. Let $i: M_{2} \rightarrow \Omega_{2}^{3} S^{3}$ be the inclusion. Clearly $H_{2}\left(\Omega_{2}^{3} S^{3} ; \mathbf{Z}_{2}\right)=\mathbf{Z}_{2} \oplus \mathbf{Z}_{2}$ and the basis of $Q_{1}[1]^{2} *[-2]$ and $Q_{2}[1]$. By Theorem $3, H_{2}\left(M_{2} ; \mathbf{Z}_{2}\right)=\mathbf{Z}_{2} \oplus \mathbf{Z}_{2}$. Note that $z_{1}^{2}, Q_{2}[1], z_{2} *$ [1] are elements of $\mathrm{H}_{2}\left(M_{2} ; \mathbf{Z}_{2}\right)$. But

$$
\left\{\begin{array}{l}
i_{*} z_{1}=Q_{1}[1] *[-1] \\
i_{*} z_{2}=Q_{2}[1] *[-1]
\end{array}\right.
$$

are given in [1, Theorem 8.6]. Hence $i_{*} z_{1}^{2}=\left(Q_{1}[1] *[-1]\right)^{2}=Q_{1}[1]^{2} *[-2]$ and $i_{*} Q_{2}[1]=Q_{2}[1]$. Therefore $i_{*}: H_{2}\left(M_{2} ; \mathbf{Z}_{2}\right) \rightarrow H_{2}\left(\Omega_{2}^{3} S^{3} ; \mathbf{Z}_{2}\right)$ is an isomorphism. But $i_{*}\left(z_{2} *[1]\right)=\left(Q_{2}[1] *[-1]\right) *[1]=Q_{2}[1]$ by $(\mathrm{A})$. Therefore $z *_{2}[1]=Q_{2}[1]$ holds.

(ii) Proof of $z *_{*} 3[1]=Q_{3}[1]$. Let $f: \mathrm{SO}(3) \rightarrow M_{2}$ be the composite of $\mathrm{SO}(3) \rightarrow M_{1} \times 1 \rightarrow M_{1} \times M_{1} \stackrel{*}{\rightarrow} M_{2}$ and let $g: \mathrm{SO}(3) \rightarrow M_{2}$ be the composite of

$$
\mathrm{SO}(3) \rightarrow S^{3} \times \mathrm{z}_{2} 1 \times 1 \rightarrow S^{3} \times \mathrm{z}_{2} M_{1} \times M_{1} \stackrel{\theta}{\rightarrow} M_{2} .
$$

Clearly $f_{*} z_{i}=z_{i} *[1]$ and $g_{*} z_{i}=Q_{i}[1]$ hold for $i=1,2,3$. But we have shown the following.

$$
f_{*} z_{1}=g_{*} z_{1}, \quad f_{*} z_{2}=g_{*} z_{2} .
$$

By Theorem 3, all we need is to prove the following equalities.

$$
\left\langle u^{3}, f_{*} z_{3}\right\rangle=\left\langle u^{3}, g_{*} z_{3}\right\rangle, \quad\left\langle u v, f_{*} z_{3}\right\rangle=\left\langle u v, g_{*} z_{3}\right\rangle .
$$

Let $\Delta$ be the diagonal; then we easily see the following.

$$
\Delta_{*} z_{3}=z_{3} \otimes 1+z_{2} \otimes z_{1}+z_{1} \otimes z_{2}+1 \otimes z_{3} .
$$

Then $\left\langle u^{3}, f_{*} z_{3}\right\rangle=\left\langle u^{2}, f_{*} z_{2}\right\rangle\left\langle u, f_{*} z_{1}\right\rangle=\left\langle u^{2}, g_{*} z_{2}\right\rangle\left\langle u, g_{*} z_{1}\right\rangle=\left\langle u^{3}, g_{*} z_{3}\right\rangle$.

$\left\langle u v, f_{*} z_{3}\right\rangle=\left\langle u v, g_{*} z_{3}\right\rangle$ is similarly proved.

\section{ACKNOWLEDGMENT}

The author is grateful to A. Kono and M. Tezuka for many useful comments.

\section{REFERENCES}

1. C. Boyer and B. Mann, Homology operations on instantons, J. Differential Geom. 28 (1988), 423-465.

2. F. Cohen, J. Lada, and P. May, The homology of iterated loop spaces, Lecture Notes in Math., vol. 533, Springer-Verlag, Berlin and New York, 1976.

3. A. Hattori, Topology of the moduli space of $\mathrm{SU}(2)$-instantons with instanton number $2, \mathrm{~J}$. Fac. Sci. Univ. Tokyo 34 (1987), 741-761.

4. __ Corrections to Topology of the moduli space of SU(2)-instantons with instanton number 2 , J. Fac. Sci. Univ. Tokyo 36 (1989), 387-388.

Department of Mathematics, Faculty of Science, University of Tokyo, Hongo, Tokyo 113 JAPAN 